\title{
High level expression of the anti-retroviral protein APOBEC3G is induced by influenza $A$ virus but does not confer antiviral activity Eva-K Pauli ${ }^{1}$, Mirco Schmolke ${ }^{1}$, Henning Hofmann ${ }^{2}$, Christina Ehrhardt ${ }^{1}$, Egbert Flory ${ }^{3}$, Carsten Münk² and Stephan Ludwig*1
}

\author{
Address: ${ }^{1}$ Institute of Molecular Virology (IMV), Centre of Molecular Biology of Inflammation (ZMBE), Westfaelische-Wilhelms-University \\ Muenster, Münster, Germany, ${ }^{2}$ Clinic for Gastroenterology, Hepatology and Infectiology, Heinrich-Heine-University, Duesseldorf, Germany and \\ ${ }^{3}$ Paul-Ehrlich-Institute (PEI), Langen, Germany \\ Email: Eva-K Pauli - evapauli@uni-muenster.de; Mirco Schmolke - mirco.schmolke@uni-muenster.de; \\ Henning Hofmann - Henning.Hofmann@med.uni-duesseldorf.de; Christina Ehrhardt - ehrhardc@uni-muenster.de; \\ Egbert Flory - floeg@pei.de; Carsten Münk - Carsten.Muenk@med.uni-duesseldorf.de; Stephan Ludwig* - ludwigs@uni-muenster.de \\ * Corresponding author
}

\section{Published: 16 April 2009}

Retrovirology 2009, 6:38 doi:10.1186/1742-4690-6-38
Received: 31 October 2008

Accepted: 16 April 2009

This article is available from: http://www.retrovirology.com/content/6/1/38

(C) 2009 Pauli et al; licensee BioMed Central Ltd.

This is an Open Access article distributed under the terms of the Creative Commons Attribution License (http://creativecommons.org/licenses/by/2.0), which permits unrestricted use, distribution, and reproduction in any medium, provided the original work is properly cited.

\begin{abstract}
Human APOBEC3G is an antiretroviral protein that was described to act via deamination of retroviral cDNA. However, it was suggested that APOBEC proteins might act with antiviral activity by yet other mechanisms and may also possess RNA deamination activity. As a consequence there is an ongoing debate whether APOBEC proteins might also act with antiviral activity on other RNA viruses. Influenza A viruses are single-stranded RNA viruses, capable of inducing a variety of antiviral gene products. In searching for novel antiviral genes against these pathogens, we detected a strong induction of $A P O B E C 3 G$ but not $A P O B E C 3 F$ gene transcription in infected cells. This upregulation appeared to be induced by the accumulation of viral RNA species within the infected cell and occurred in an NF- $\kappa B$ dependent, but MAP kinase independent manner. It further turned out that $A P O B E C$ expression is part of a general IFN $\beta$ response to infection. However, although strongly induced, $A P O B E C 3 G$ does not negatively affect influenza $A$ virus propagation.
\end{abstract}

\section{Findings}

In patients infected with HIV-1, the expression of human apolipoprotein (apo) B mRNA editing enzyme catalytic polypeptide 1-like protein 3G (APOBEC3G) was observed to be elevated [1], although this was not confirmed in cell culture experiments [2,3]. Members of the APOBEC3 family are known to act with anti-retroviral activity against HIV $[4,5]$, but they also inhibit replication of hepatitis B virus (HBV) [6], and adeno-associated virus type 2 [7]. The anti-retroviral activity of human APOBEC3 proteins is probably conferred by cytidine deamination of the newly synthesized first viral cDNA strand. This mechanism is counteracted by the HIV-1 protein virion infectivity factor
(Vif) [8-12]. However, human APOBEC3 proteins may not only have anti-retroviral or anti-HBV activity. Two findings have triggered a broader interest in these proteins with regard to a potential antiviral action against RNA viruses. First, besides its DNA deamination activity, human APOBEC3 proteins were reported to also possess RNA deamination activity [13]. Second, DNA deamination activity may not be the only antiviral action of these proteins [13-16] suggesting that APOBEC3s might possess functions that render them effective against other viruses, which do not have any DNA-intermediates during replication such as influenza A virus. 
In global gene expression profiling studies of influenza A virus-infected cells, we observed strongly elevated transcription of human APOBEC3G. This finding was verified by quantitative Real-time PCR (qRT-PCR) [17] with specific primers against APOBEC3G and APOBEC3F (Figure 1A) [18-20] in lung epithelial cells (A549) (Figure 1B) and in primary human endothelial cells (HUVEC) (Figure 1C) infected with the human influenza virus A/Puerto Rico/8/ 34 H1N1 (PR8). The upregulation of APOBEC3G was also confirmed on protein level as determined in Western blot analysis (Figure 1E). Protein expression steadily increased with time up to 16 hours post infection but dropped again at 24 hours p.i (Figure 1E) most likely due to host cell protein shut-off induced by the virus. Interestingly, such an upregulation of human APOBEC3G transcription was not reported for cells infected with HIV-1 [2,3], although higher expression levels of human APOBEC3G in HIV-1 infected patients is described in the literature [1]. Upregulation of APOBEC3G was also confirmed in cells infected with the human H5N1 influenza virus isolate A/Thailand/ (KAN-1)/2004 (H5N1) (data not shown), suggesting that transcriptional induction of APOBEC3G is a general phenomenon in influenza A virus infected cells. Interestingly, the paralogue human APOBEC3F was not found to be upregulated in A549 cells and was only marginally induced in HUVEC (Figure 1B and 1C). This is noteworthy, since human APOBEC3F and human APOBEC3G share more than $90 \%$ promoter sequence similarity and appear to be transcriptionally co-regulated $[4,5]$. However, co-regulated induction of expression was not observed in our experiments. Instead we found that the mRNA copy number of APOBEC3F remains at a constant high level in uninfected and infected A549 cells, while the copy numbers of APOBEC3G are at a low level in uninfected cells and rise upon viral infection (Figure 1D), suggesting distinct transcriptional regulation despite high promoter sequence similarity.

Given the particular strong induction of human APOBEC3G in influenza A virus-infected cells, we addressed the question which virus-induced intracellular signalling pathways are required for human APOBEC3G mRNA transcription. Influenza virus infection induces a variety of signalling pathways such as the Raf/MEK/ERK kinase cascade, the p38 signalling pathway and the IKK/ NFKB pathway $[21,22]$. PMA, an effective inducer of the classical Raf/MEK/ERK cascade, has been reported to induce human APOBEC3G gene expression in $\mathrm{H} 9$ cells via PKC [23]. However, in the cell types used in our study, PMA (100-200 ng/ml) was only a weak inducer of APOBEC3G expression, and inhibition of the Raf/MEK/ ERK cascade by the MEK inhibitor U0126 $(2-10 \mu \mathrm{g} / \mathrm{ml})$ did not result in reduced human APOBEC3G mRNA levels in virus-infected A549 cells (data not shown). Activation of the p38 signalling cascade by virus infection involves the phosphorylation of $\mathrm{p} 38$ by the MAP kinase kinase, MKK6. To block the pathway at this level of the cascade we overexpressed a dominant negative mutant of MKK6 (MKK6Ala) that was previously shown to efficiently suppress the activation of p38 [24]. Successful transduction of the retroviral vector pEGZ-MKK6Ala was monitored by FACS-analysis of GFP (data not shown) that is expressed from a second reading frame of the mRNA of the transgene [24]. Inhibition of the $\mathrm{p} 38$ phosphorylation by either stable overexpression of the dominant-negative form of MKK6 (Figure 2A) or application of the p38 inhibitor SB203580 $(20 \mu \mathrm{M})$ (data not shown) did not affect the induced transcription of APOBEC3G. These findings argue against a prominent role of either, ERK or p38 MAPK cascade in viral APOBEC3G induction.

The IKK2/NF-кB module is another influenza virus-activated signalling cascade that is known to regulate a variety of genes. This includes IFN $\beta$ transcription, which is controlled by an enhanceosome, composed of the transcription factors IRF3/7, NF- $\kappa \mathrm{B}$, and AP-1 [22]. To assess the involvement of IKK2 and NF- $\mathrm{B}$ in virus-induced APOBEC3G expression, we used A549 cells that were retrovirally transduced with the vector pEGZ-IKK2KD. This transduction allows for the stable expression of the dominant negative mutant of IKB kinase 2 (IKK2), an approach that has been successfully used previously to efficiently blunt NF- $\kappa B$ activity $[25,26]$. Upon infection of these mutant-expressing cells, APOBEC3G mRNA levels were reduced compared to control cells (Figure 2A) to a similar extent that was observed for the IFN $\beta$ gene (Figure $2 \mathrm{~B})$. The same pattern of APOBEC3G expression was also observed in infected cells pre-treated with the NF- $\mathrm{KB}$

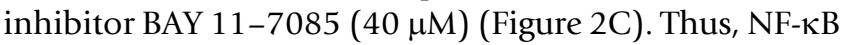
activity appeared to be crucial for viral APOBEC3G induction.

To independently analyse whether NF- $\kappa \mathrm{B}$ might play a role in APOBEC3G induction, we stimulated cells with TNF $\alpha(20 \mathrm{ng} / \mathrm{ml})$, a very strong activator of NF-kB [27]. However, TNFa stimulation did not result in enhanced APOBEC3G gene transcription (data not shown), indicating that NF- $\mathrm{KB}$ activity alone is not sufficient to induce human APOBEC3G gene transcription.

Influenza virus infection results in type I IFN production (Figure 2B) and subsequent expression of IFN-responsive genes [28-30]. So far, it was not clear from the literature whether human APOBEC3 genes are induced by type I IFNs. While IFN-dependency was reported for the hepatoma cell lines HepG2 and Huh7 [18,31] and for macrophages [32], human APOBEC3 proteins are not inducible in H9 cells by type I and type II IFN [23]. To specifically address this issue for the lung epithelial cell line used in our study, A549 cells were incubated for different 
A

human APOBEC3F primer

\begin{tabular}{|l|l|l|l|l|l|l|}
\hline Plasmid copy & \multicolumn{3}{|c|}{ hA3F } & \multicolumn{3}{c|}{ hA3G } \\
\hline number & $10^{6}$ & $10^{4}$ & $10^{2}$ & $10^{6}$ & $10^{4}$ & $10^{2}$ \\
\hline CT-value & 21.5 & 28.7 & 32.8 & $>40$ & $>40$ & $>40$ \\
\hline
\end{tabular}

human APOBEC3G primer

\begin{tabular}{|l|l|l|l|l|l|c|}
\hline Plasmid copy & \multicolumn{3}{|c|}{ hA3F } & \multicolumn{3}{c|}{ hA3G } \\
\hline number & $10^{6}$ & $10^{4}$ & $10^{2}$ & $10^{6}$ & $10^{4}$ & $10^{2}$ \\
\hline CT-value & $>40$ & $>40$ & $>40$ & 21.5 & 28.2 & $>40$ \\
\hline
\end{tabular}
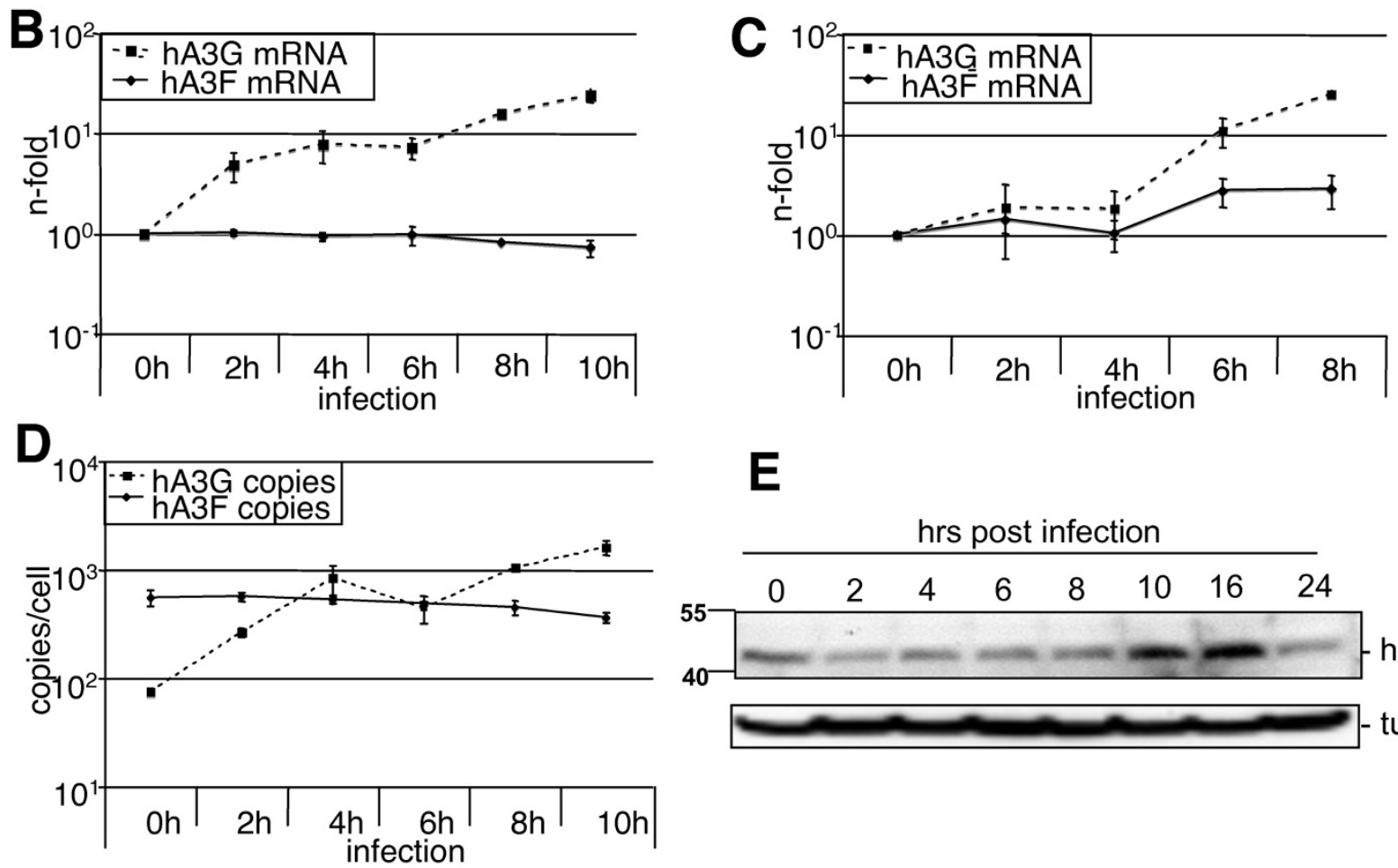

E

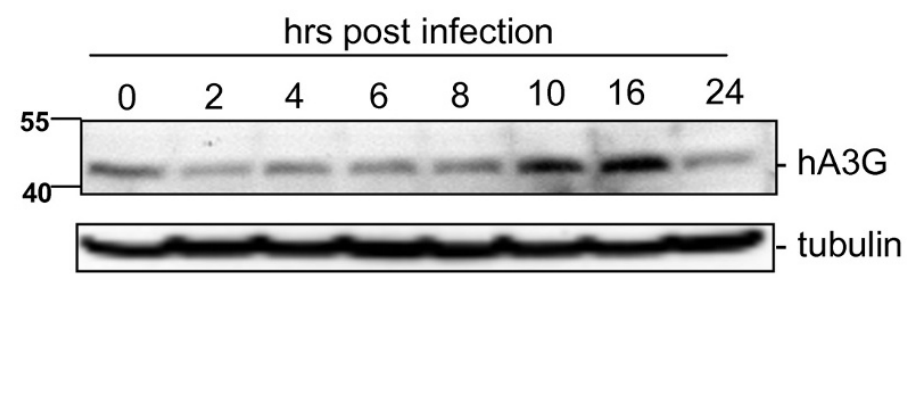

Figure I (see legend on next page) 
Figure I (see previous page)

Virus-induced human APOBEC3G gene transcription. (A) Determination of the binding specificity of human APOBEC3F and human APOBEC3G primers in quantitative real time PCR ( $R$ RT-PCR). Serial dilutions of the C-terminally HAtagged plasmids pcDNA_huAPOBEC3F (hA3F) (described by $\mathrm{H}$. Muckenfuss and colleagues) or pcDNA_huAPOBEC3G (generously provided by Nathaniel R. Landau) were analysed using either human APOBEC3F or APOBEC3G specific primer pairs in QRT-PCR. The copy number of each plasmid and the corresponding CT-values (cycle number of the first detectable signal) are given. Low CT-values indicate high amounts of the DNA-sequence of interest. CT-values above 30 are commonly considered as non-specific signals. The qRT-PRC-program is limited to 40 cycles; due to that fact that CT-values $>40$ indicate undetectable amounts of DNA. (B-E) The influenza virus strain A/Puerto Rico/8/34 HINI (PR8) was diluted in PBS containing 0.6\% sterile BSA and I\% penicillin/streptomycin and used to infect A549 cells (B and D-E) or HUVEC $(C)(M O I=5)$ for the time points indicated. At two-hourly intervals post infection, RNA was isolated using the RNeasy mini-kit (Qiagen), and $3 \mu g$ of total RNA were transcribed into cDNA using $0.5 \mu \mathrm{g}$ oligo dT-primer (16 mer) and $200 \mathrm{U}$ Revert Aid $\mathrm{H}^{-}$(Fermentas) according to the manufacture's protocol. mRNA levels of IFN $\beta$, human APOBEC3F and human APOBEC3G were assessed by qRT-PCR using primer pairs for human APOBEC3F and 3G; or for human IFN $\beta$ as follows: IFN $\beta$ fwd 5'-GGC CAT GAC CAA CAA GTG TCT CCT CC-3' and IFN $\beta$ rev 5'-GCG CTC AGT TTC GGA GGT AAC CTG T-3'. Induced transcription of mRNA was calculated as n-fold using GAPDH as reference gene. (D) Determination of the copy numbers per cell of human APOBEC3F or human APOBEC3G. (E) Infected A549 cells were lysed at the time points indicated. Endogenous expression of human APOBEC3G was determined with the hA3G specific antibody ApoCI7 (NIH AIDS Research and Reference Reagent Program) in Western blots. An anti-tubulin (B5-I-2, Sigma) blot served as a loading control.

time periods with recombinant IFN $\beta(100 \mathrm{U} / \mathrm{ml})(\mathrm{PBL})$, and the levels of human APOBEC3G and human APOBEC3F mRNAs were determined by qRT-PCR (Figure 2D).

IFN $\beta$ stimulation led to a nearly 20 -fold induction of the human APOBEC3G mRNA (Figure 2D), which could also be observed at the protein level (Figure 2E); by contrast, the human APOBEC3F mRNA was not affected at all (Figure 2D). Strikingly, this pattern of human APOBEC3G versus human APOBEC3F expression exactly matched the results obtained upon virus infection (Figure 1B). This suggests that IFN $\beta$, expressed upon virus infection in an $\mathrm{NF}-\kappa \mathrm{B}$ dependent manner, may be an indirect trigger of human APOBEC3G expression, leaving still open the question about the initial viral inducer.

IFN $\beta$ transcription in infected cells is known to be mainly induced by single-stranded or partially double-stranded RNA. Such RNA species accumulate during infection within the host cell and serve as a pathogen pattern sensed by cells $[33,34]$. To examine whether different RNA species serve as inducer of APOBEC3G gene expression, total RNAs isolated from influenza virus infected ("viral RNA") or uninfected cells ("cellular RNA"), or the dsRNA analogue poly (I:C), or short ssRNA bearing a 5'-triphosphate were used as stimuli to elicit a gene response. These RNAs were transfected into A549 cells, and mRNA levels of human APOBEC3G and IFN $\beta$ were determined (Figure 3A-D). While transfection of RNA from uninfected cells led to no significant gene induction, RNA from virally infected cells resulted in upregulation of both, human APOBEC3G and IFN $\beta$ transcription (Figure 3A and 3B). Stimulation using either poly (I:C) or 5'-triphosphate
RNA led to even a stronger induction of APOBEC3G (Figure $3 \mathrm{C}$ and $3 \mathrm{D}$ ). In summary, our findings indicate that human APOBEC3G is induced upon viral infection as a part of the antiviral response mediated by type I IFN. This response is triggered by the recognition of different RNA species by distinct receptors such as TLR3, RIG-I and/or MDA-5. Interestingly, we did not observe any human APOBEC3F induction, neither upon viral infection nor with IFN $\beta$ stimulation (Figure $1 \mathrm{~B}$ and $2 \mathrm{D}$ ), albeit both promoters carry ISRE elements [35]. Thus, we hypothesized that human APOBEC3G may be selectively induced and may confer a specific antiviral activity in influenza virus infected cells.

To test this assumption we first transiently over expressed HA-tagged human APOBEC3G (Figure 4B) and assessed the efficiency of viral propagation in these cells. Surprisingly, in the presence of human APOBEC3G, progeny virus titres were slightly elevated compared to the vector control (Figure 4A, white bars). This correlated with a slightly higher expression level of the viral polymerase subunit PB1 (Figure 4B). To circumvent potential transient transfection artefacts and to enhance the number of transgene-expressing cells, we generated cell lines, stably expressing human APOBEC3G (Figure 4C and 4D). After selection of stably APOBEC3G expressing cells by antibiotic treatment, the cells were infected with different influenza A virus strains at various multiplicities of infection (MOI) (Figure 4A, grey and black bars). In contrast to the transient situation, viral propagation was not affected in these stably transfected cells, although the transgene was expressed well in MDCK cells (Figure 4C) as well as in A549 cells (Figure 4D). Thus, although influenza A virus induces human APOBEC3G transcription in an NF- $\kappa$ B 
A
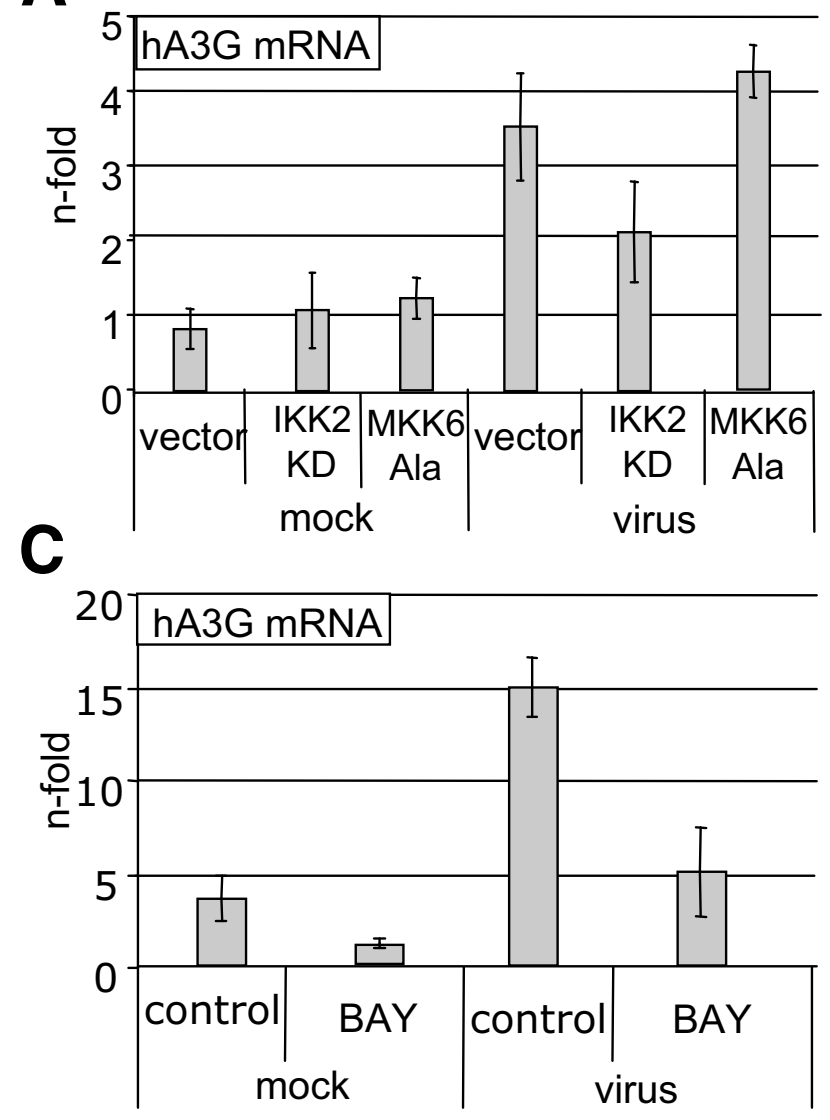

$\mathbf{B}_{10}$
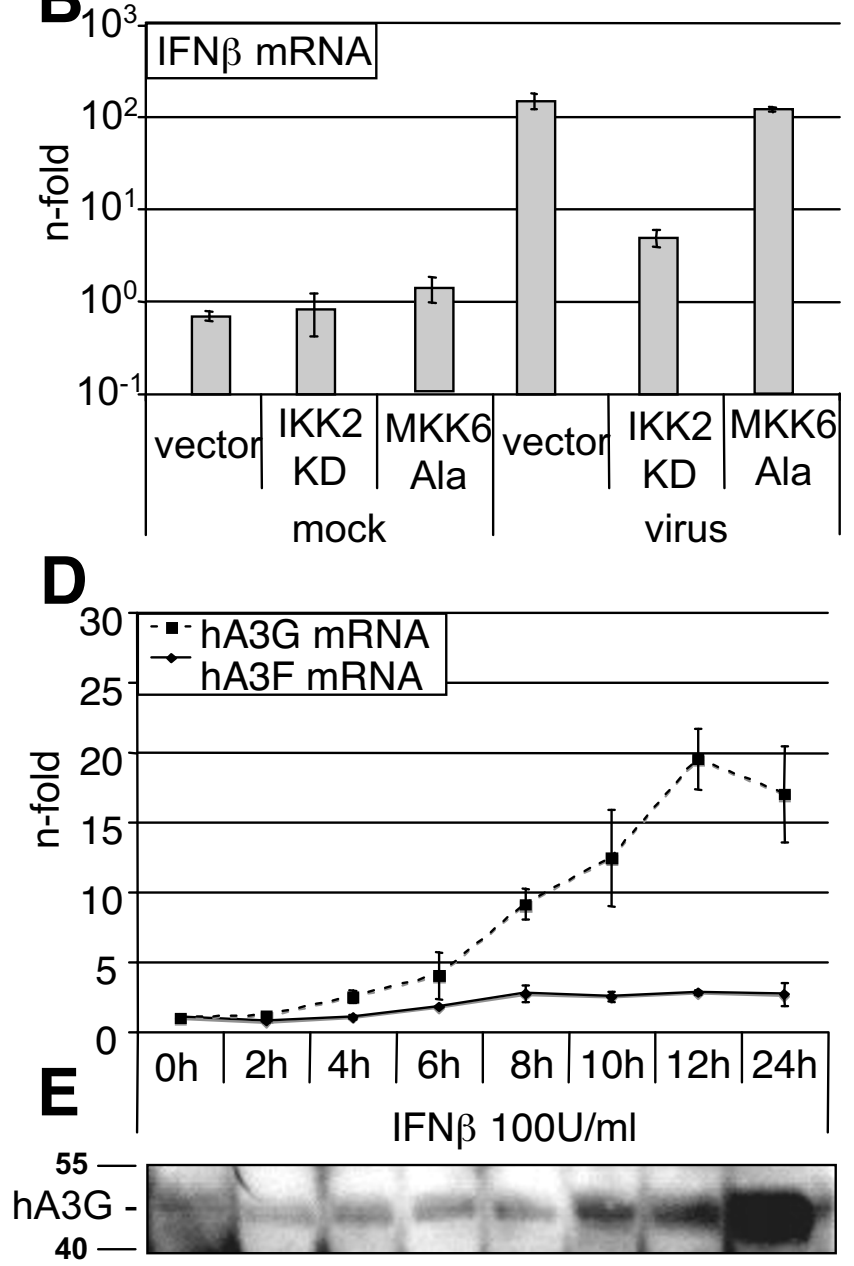

Figure 2

IFN $\beta$-induced transcription of human APOBEC3G. (A and B) A549 cells stably overexpressing the dominant negative mutants IKK2KD or MKK6Ala. These mutant kinases were cloned in the retroviral pEGZ-vector. In this vector GFP is expressed by an internal ribosomal entry site (IRES) from the same mRNA as the IKK2KD and MKK6Ala transgenes, allowing transgene expression to be monitored by FACS-analysis (data not shown). MKK6Ala and IKK2KD overexpressing cells were infected for 10 hours with the influenza A virus strain PR8 $(\mathrm{MOI}=5)$. Following infection, RNA was isolated with the RNeasy mini kit (Qiagen), reverse transcribed as described above, and cDNA was subjected to qRT-PCR. (C) A549 cells were pretreated for 30 minutes with the NF- $\kappa B$ specific inhibitor BAY I I-7085 (40 $\mu \mathrm{M})$ before infection with PR8 (MOI = 5) for I0 hours. RNA was subjected to qRT-PCR. The mRNA levels of human APOBEC3G (A and C) or IFN $\beta$ (B) were assessed by qRT-PCR. (D) A549 cells were stimulated with IFN $\beta$ (I00 U/ml) (Invitrogen) for the time points indicated. The mRNA levels of human APOBEC3F and human APOBEC3G were determined by means of qRT-PCR. Induction of gene transcription was calculated as n-fold of untreated cells, which was arbitrarily set as I, as described by Livak and Schmittgen. (E) A549 cells were stimulated as in (D), and cell lysates were subjected to Western blots using the hA3G specific antibody ApoCI7 (NIH)

and IFN $\beta$ dependent manner, the forced expression of human APOBEC3G did not result in any antiviral effect on this virus. This is different from the situation with HIV1. APOBEC3G shows antiviral activity against HIV-1 and other retroviruses $[8,2]$. However, HIV-1 does not induce APOBEC3G transcription in cell culture $[2,3]$. In support of a specific rather than broad antiviral activity of
APOBEC3G, Kremer et al. [36] had reported that overexpressed human APOBEC3G also has no antiviral effect against vaccinia virus (VACV). In summary, we conclude that human APOBEC3G is induced by influenza A viral RNA, via an NF- $\mathrm{B}$ B dependent mechanism as part of the antiviral IFN response program but does not exhibit an antiviral effect against influenza A virus. 

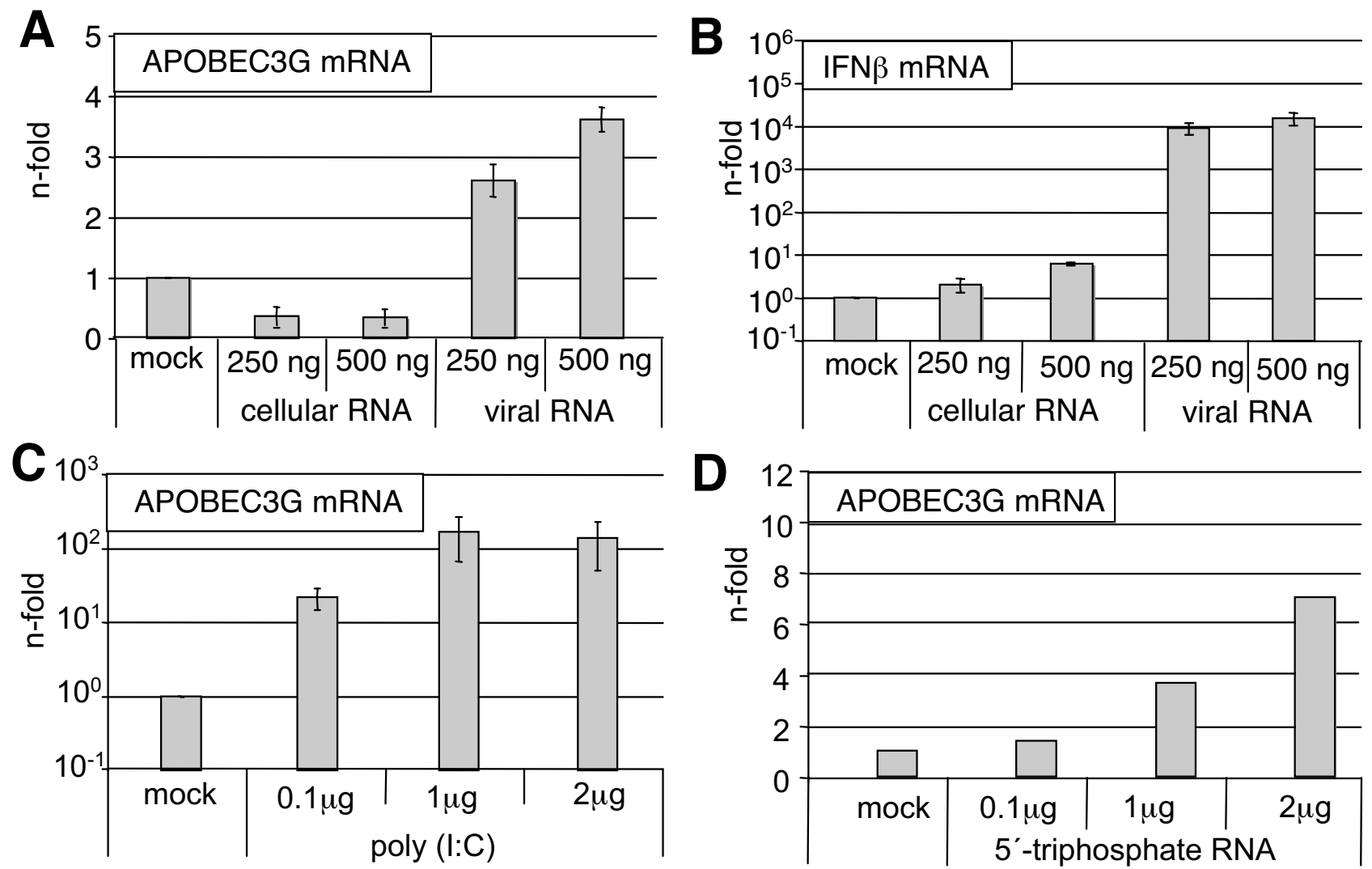

\section{Figure 3}

Induction of human APOBEC3G mRNA by different RNA species. (A and B) A549 cells (I.5 × 106/6 cm dish) were transfected with indicated amounts of total RNA from virally infected ("viral RNA") or uninfected ("cellular RNA") A549 cells. RNA from uninfected or virally infected cells was generated by isolation of total RNA from cells either infected with PR8 (MOI $=5$ ) or from uninfected cells using the Qiagen RNeasy kit according to the manufacturer's instructions. For stimulation, these RNAs were transfected for 10 hours using Lipofectamine 2000 (L2000) (Invitrogen) according to the manufacturer's protocol. (C) A549 cells were transfected with either different amounts of poly (l:C) (Amersham Biosciences) or with 5'-triphosphate RNA (D). 5'-triphosphate RNA was generated by reverse transcription of a short PCR product using the MEGAshortscript kit (Ambion) according to the manufacturer's instructions. mRNA levels of IFN $\beta$ (B) or human APOBEC3G (A, C and D) were determined by qRT-PCR as described. Levels of gene induction were calculated as n-fold of the background of untreated cells, which was arbitrarily set as $I$. 


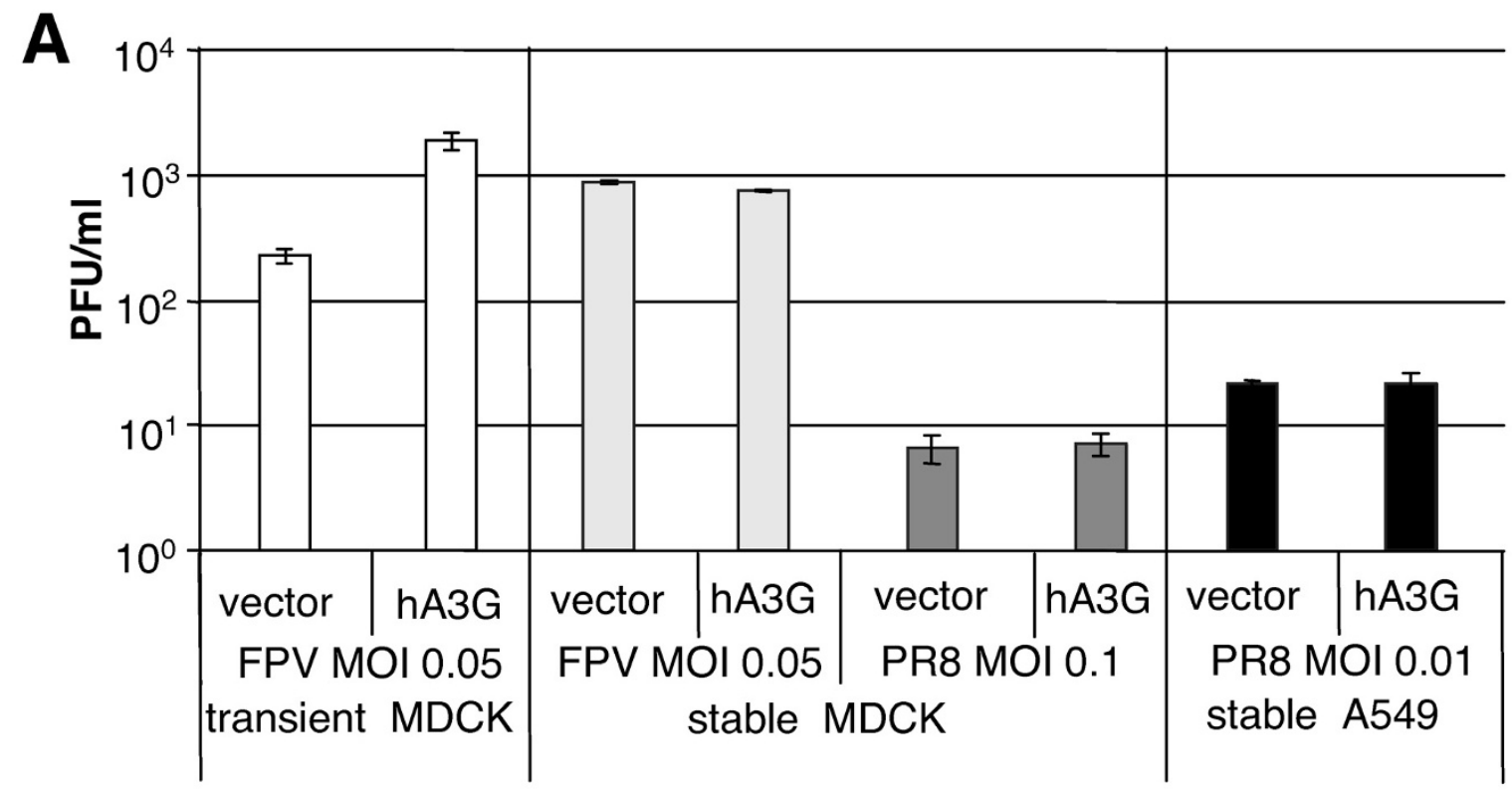

B
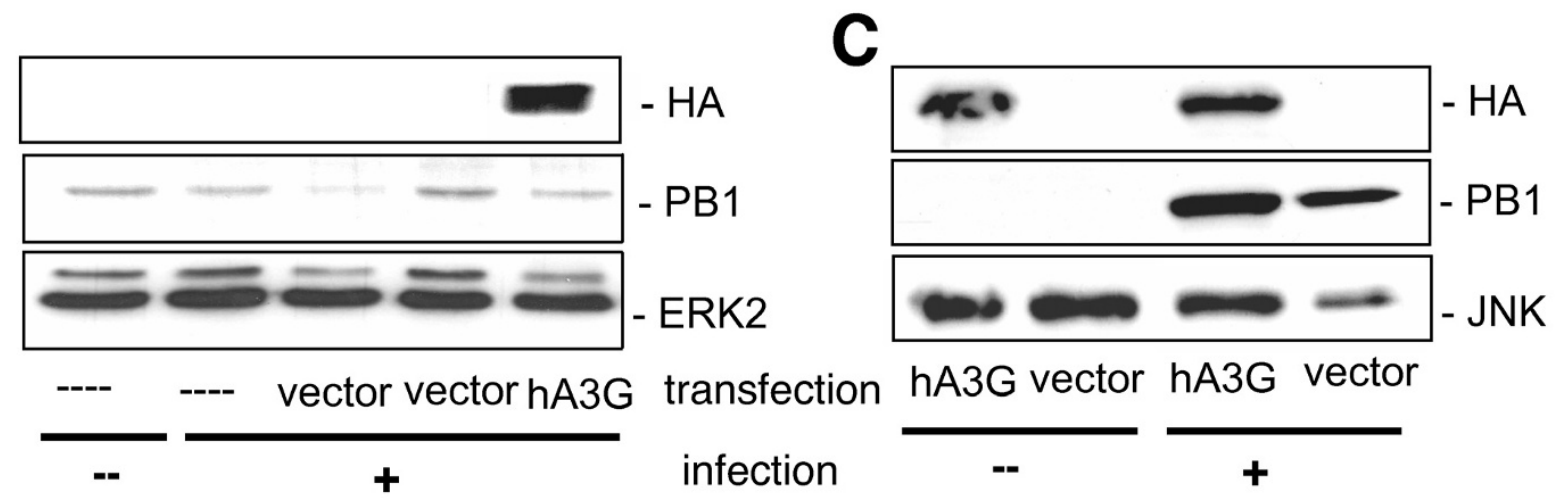

D

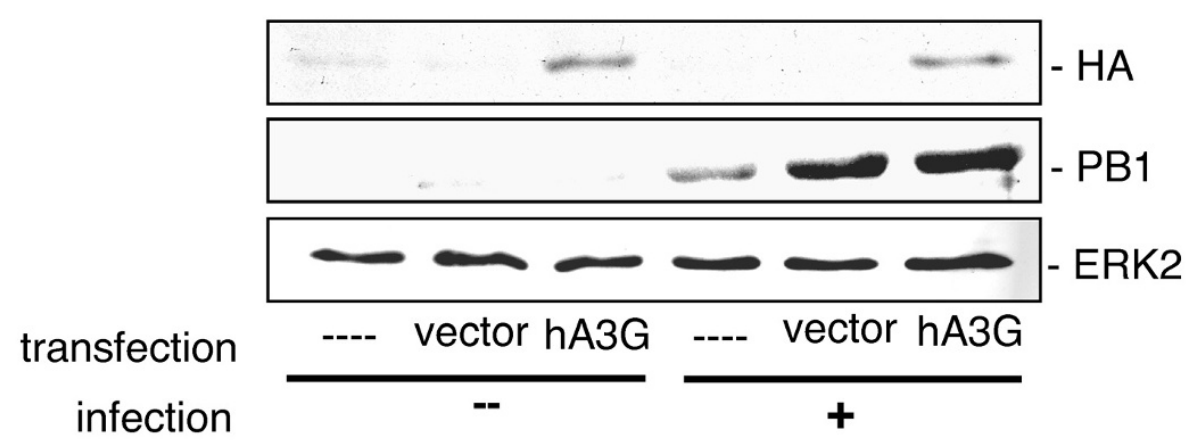

Figure 4 (see legend on next page) 
Figure 4 (see previous page)

Influence of the human APOBEC3G protein on viral replication. (A) Transiently (white bars) or stably human APOBEC3G transfected (light and dark grey bars) MDCK cells were infected with avian influenza virus A/FPV/Bratislava/79 $(\mathrm{H7N7})(\mathrm{FPV})(\mathrm{MOI}=0.05)$ for 9 hours or A/Puerto Rico/8/34 HINI (PR8) $(\mathrm{MOI}=0 . \mathrm{I})$ virus for 16 hours. Both virus strains were originally taken from the collection of the Institute of Virology, University of Giessen, Germany. Stably transfected A549 cells (black bars) were infected with PR8 for 16 hours $(\mathrm{MOI}=0.0 \mathrm{I})$. Supernatants were taken, and virus titres were determined by means of plaque assay. (B and C) MDCK cells or (D) A549 cells were transfected with $3 \mu \mathrm{g}$ DNA/6 well dish pcDNAAPOBEC3G or pcDNA3.I empty vector using L2000 (Invitrogen) according to manufacturer's instructions. To generate a bulk amount of stable expressing cells, MDCK cells (C) or A549 cells (D) were treated with G4l8 $300 \mu \mathrm{g} / \mathrm{ml}$ for selection of APOBEC3G expression for four weeks. Thereafter, cells were infected with FPV at $\mathrm{MOI}=0.05(\mathrm{~B})$ or at $\mathrm{MOI}=0 . \mathrm{I}$ for 9 hours (C) or with PR8 at $\mathrm{MOI}=0.00 \mathrm{I}$ for 16 hors (D). Expression of HA-tagged human-APOBEC3G was detected by anti-HA 3FI0 (Roche) antibody. To control equivalent protein loading, ERK2 or JNK were detected by anti-ERK2 (Santa Cruz) or antiJNK antibody (Santa Cruz). Viral replication was monitored by detection of the viral polymerase protein using an anti-PBI antibody (Santa Cruz).

\section{Competing interests}

The authors declare that they have no competing interests.

\section{Authors' contributions}

EKP, MS, CE and HH have performed experimental work, contributed data and gave conceptual input in the study design. EF and CM have provided important material and have been involved in drafting the manuscript and revising it critically for important intellectual content. SL has designed and has guided the study, interpreted the data and wrote the manuscript.

\section{Acknowledgements}

The following reagent was obtained through the NIH AIDS Research and Reference Reagent Program, Division of AIDS, NIAID, NIH: anti-ApoCI7 from Dr. Klaus Strebel. This work was supported by several grants from the Deutsche Forschungsgemeinschaft (DFG) (Lu477-I I/2, SFB293 AI7, Graduate School GRK I409), the Interdisciplinary Clinical Research Centre (IZKF) of the University of Münster, the FluResearchNet funded by the German Ministry of Education and Research (BMBF), and the EC funded STREP EUROFLU.

\section{References}

I. Ulenga NK, Sarr AD, Thakore-Meloni S, Sankale JL, Eisen G, Kanki PJ: Relationship between human immunodeficiency type I infection and expression of human APOBEC3G and APOBEC3F. J Infect Dis 2008, 198:486-492.

2. Stopak K, de Noronha C, Yonemoto W, Greene WC: HIV-I Vif blocks the antiviral activity of APOBEC3G by impairing both its translation and intracellular stability. Mol Cell 2003, | 2:59|-60|

3. Mehle A, Strack B, Ancuta P, Zhang C, McPike M, Gabuzda D: Vif overcomes the innate antiviral activity of APOBEC3G by promoting its degradation in the ubiquitin-proteasome pathway. $\quad J$ Biol Chem 2004, 279:7792-7798.

4. Bishop KN, Holmes RK, Sheehy AM, Davidson NO, Cho SJ, Malim $\mathrm{MH}$ : Cytidine deamination of retroviral DNA by diverse APOBEC proteins. Curr Biol 2004, I4:1392-1396.

5. Wiegand HL, Doehle BP, Bogerd HP, Cullen BR: A second human antiretroviral factor, $A P O B E C 3 F$, is suppressed by the HIVI and HIV-2 Vif proteins. EMBO J 2004, 23:245 I-2458.

6. Turelli $P$, Mangeat B, Jost S, Vianin S, Trono D: Inhibition of hepatitis B virus replication by APOBEC3G. Science 2004, 303:1829.

7. Chen H, Lilley CE, Yu Q, Lee DV, Chou J, Narvaiza I, Landau NR Weitzman MD: APOBEC3A is a potent inhibitor of adenoassociated virus and retrotransposons. Curr Biol 2006, 16:480-485
8. Sheehy AM, Gaddis NC, Choi JD, Malim MH: Isolation of a human gene that inhibits HIV-I infection and is suppressed by the viral Vif protein. Nature 2002, 4I 8:646-650.

9. Harris RS, Bishop KN, Sheehy AM, Craig HM, Petersen-Mahrt SK Watt IN, Neuberger MS, Malim MH: DNA deamination mediates innate immunity to retroviral infection. Cell 2003, I | 3:803-809.

10. Mangeat B, Turelli P, Caron G, Friedli M, Perrin L, Trono D: Broad antiretroviral defence by human APOBEC3G through lethal editing of nascent reverse transcripts. Nature 2003 , 424:99- 103

II. Goila-Gaur R, Strebel K: HIV-I Vif, APOBEC, and intrinsic immunity. Retrovirology 2008, 5:5I.

12. Pillai SK, Wong JK, Barbour JD: Turning up the volume on mutational pressure: is more of a good thing always better? (A case study of HIV-I Vif and APOBEC3). Retrovirology 2008, 5:26.

13. Bishop KN, Holmes RK, Sheehy AM, Malim MH: APOBEC-mediated editing of viral RNA. Science 2004, 305:645.

14. Newman EN, Holmes RK, Craig HM, Klein KC, Lingappa JR, Malim $\mathrm{MH}$, Sheehy AM: Antiviral function of APOBEC3G can be dissociated from cytidine deaminase activity. Curr Biol 2005, 15:166-170.

15. Shindo K, Takaori-Kondo A, Kobayashi M, Abudu A, Fukunaga K, Uchiyama T: The enzymatic activity of CEMI5/Apobec-3G is essential for the regulation of the infectivity of HIV-I virion but not a sole determinant of its antiviral activity. J Biol Chem 2003, 278:444I2-444I6.

16. Cho SJ, Drechsler H, Burke RC, Arens MQ, Powderly W, Davidson NO: APOBEC3F and APOBEC3G mRNA levels do not correlate with human immunodeficiency virus type I plasma viremia or CD4+ T-cell count. J Virol 2006, 80:2069-2072.

17. Livak KJ, Schmittgen TD: Analysis of relative gene expression data using real-time quantitative PCR and the 2(-delta delta C(T)) method. Methods 200I, 25:402-408.

18. Tanaka $Y$, Marusawa H, Seno H, Matsumoto $Y$, Ueda $Y$, Kodama $Y$, Endo Y, Yamauchi J, Matsumoto T, Takaori-Kondo A, Ikai I, Chiba T: Anti-viral protein APOBEC3G is induced by interferonalpha stimulation in human hepatocytes. Biochem Biophys Res Commun 2006, 341:314-319.

19. Muckenfuss H, Hamdorf M, Held U, Perkovic M, Löwer J, Cichutek K, Flory E, Schumann GG, Münk C: APOBEC3 proteins inhibit human LINE-I retrotransposition. I Biol Chem 2006, 28I:22 I6I-22I72.

20. Mariani R, Chen D, Schröfelbauer B, Navarro F, König R, Bollmann B Münk C, Nymark-McMahon H, Landau NR: Species-specific exclusion of APOBEC3G from HIV-I virions by Vif. Cell 2003, I | 4:2|-3|.

21. Ludwig S: Exploited defense: how influenza viruses take advantage of antiviral signaling responses. Future Virol 2007, 2:91-100.

22. Ludwig S, Pleschka S, Planz O, Wolff $\mathrm{T}$ : Ringing the alarm bells: signalling and apoptosis in influenza virus infected cells. Cell Microbiol 2006, 8:375-386. 
23. Rose KM, Marin M, Kozak SL, Kabat D: Transcriptional regulation of APOBEC3G, a cytidine deaminase that hypermutates human immunodeficiency virus. J Biol Chem 2004, 279:41744-4I749.

24. Ludwig S, Hoffmeyer A, Moebeler M, Kilian K, Häfner H, Neufeld B, Han J, Rapp UR: The stress inducer arsenite activates mitogenactivated protein kinase extracellular signal-regulated kinases $I$ and 2 via a MAPK kinase 6/p38-dependent pathway. J Biol Chem 1998, 273:1917-1922.

25. Wurzer WJ, Ehrhardt C, Pleschka S, Berberich-Siebelt F, Wolff T, Walczak $\mathrm{H}$, Planz $\mathrm{O}$, Ludwig S: NF-kappaB-dependent induction of tumor necrosis factor-related apoptosis-inducing ligand (TRAIL) and Fas/FasL is crucial for efficient influenza virus propagation. J Biol Chem 2004, 279:3093I-30937.

26. Viemann D, Goebeler M, Schmid S, Klimmek K, Sorg C, Ludwig S, Roth J: Transcriptional profiling of IKK2/NF-kappa B- and p38 MAP kinase-dependent gene expression in TNF-alpha-stimulated primary human endothelial cells. Blood 2004 , 103:3365-3373.

27. Cao Z, Tanaka M, Regnier C, Rothe M, Yamit-hezi A, Woronicz JD, Fuentes ME, Durnin MH, Dalrymple SA, Goeddel DV: NF-kappa B activation by tumor necrosis factor and interleukin- $I$. Cold Spring Harb Symp Quant Biol 1999, 64:473-483.

28. Geiss G, Jin G, Guo J, Bumgarner R, Katze MG, Sen GC: A comprehensive view of regulation of gene expression by doublestranded RNA-mediated cell signaling. J Biol Chem 200I, 276:30|78-30|82.

29. Geiss GK, An MC, Bumgarner RE, Hammersmark E, Cunningham D, Katze MG: Global impact of influenza virus on cellular pathways is mediated by both replication-dependent and -independent events. I Virol 200I, 75:432I-433I.

30. Geiss GK, Salvatore M, Tumpey TM, Carter VS, Wang X, Basler CF, Taubenberger JK, Bumgarner RE, Palese P, Katze MG, Garcia-Sastre $A$ : Cellular transcriptional profiling in influenza $A$ virusinfected lung epithelial cells: the role of the nonstructura NSI protein in the evasion of the host innate defense and its potential contribution to pandemic influenza. Proc Natl Acad Sci USA 2002, 99: 10736-1074I.

31. Bonvin M, Achermann F, Greeve I, Stroka D, Keogh A, Inderbitzin D, Candinas D, Sommer P, Wain-Hobson S, Vartanian JP, Greeve J: Interferon-inducible expression of APOBEC 3 editing enzymes in human hepatocytes and inhibition of hepatitis $B$ virus replication. Hepatology 2006, 43: I364-1374

32. Peng G, Lei KJ, Jin W, Greenwell-Wild T, Wahl SM: Induction of APOBEC3 family proteins, a defensive maneuver underlying interferon-induced anti-HIV-I activity. J Exp Med 2006, 203:4l-46.

33. Hornung V, Ellegast J, Kim S, Brzozka K, Jung A, Kato $H$, Poeck $H$, Akira S, Conzelmann KK, Schlee M, Endres S, Hartmann G: 5'-Triphosphate RNA is the ligand for RIG-I. Science 2006, 3 I 4:994-997.

34. Pichlmair A, Schulz O, Tan CP, Naslund TI, Liljestrom P, Weber F, Reis e Sousa C: RIG-I-mediated antiviral responses to singlestranded RNA bearing 5'-phosphates. Science 2006, 3 I 4:997-100।.

35. Chen K, Huang J, Zhang C, Huang S, Nunnari G, Wang FX, Tong X, Gao L, Nikisher K, Zhang H: Alpha interferon potently enhances the anti-human immunodeficiency virus type I activity of APOBEC3G in resting primary CD4 T cells. J Virol 2006 80:7645-7657.

36. Kremer M, Suezer Y, Martinez-Fernandez Y, Munk C, Sutter G, Schnierle BS: Vaccinia virus replication is not affected by APOBEC3 family members. Virol J 2006, 3:86.
Publish with Bio Med Central and every scientist can read your work free of charge

"BioMed Central will be the most significant development for disseminating the results of biomedical research in our lifetime. "

Sir Paul Nurse, Cancer Research UK

Your research papers will be:

- available free of charge to the entire biomedical community

- peer reviewed and published immediately upon acceptance

- cited in PubMed and archived on PubMed Central

- yours - you keep the copyright
BioMedcentral 